Journal of Engineering and Applied Sciences 14 (Special Issue 7): 10067-10074, 2019

ISSN: 1816-949X

(c) Medwell Journals, 2019

\title{
Optical Properties Study of II-VI Semiconductors NPs Prepared by Laser Ablation in Liquid Technique
}

\author{
Lamis Faaz Nassir \\ College of Nursing, University of Babylon, Babylon, Iraq
}

\begin{abstract}
$\overline{\text { Abstract: The use off laser ablation to generate nanoparticle of a solid target in a liquid environment is an fast, }}$ easy and "green" method to product a large scale of nanomaterials with tailored properties. In this research we have been prepared II-VI nanoparticles by using laser ablation in liquid technique by Nd:YAG laser [pulse, width of, 9 nsec, repetition rate of $1 \mathrm{~Hz}, \lambda=1064 \mathrm{~nm}$ with different laser energy and laser fluence, respectively (60, 100, 140, $180 \mathrm{~mJ} /$ pulse), $\left(0.433,0.722,1.011,1.299, \mathrm{~J} / \mathrm{cm}^{2}\right)$. The synthesized II-VI NPs thin films of thickness $(320 \pm 10 \mathrm{~nm}$ ) have been characterized by (AFM) and UV-VIS, spectroscopy. The (AFM) images results had showed that average grain size of II-VI NPs change between of 65.42-90.38 nm. The major optical parameters like extinction coefficients $(\mathrm{k})$, absorption coefficient $(\alpha)$ and band gap have been calculated from the, transmission spectra. The probable optical transition in these films is found to be direct and allowed where band gap of II-VI NPs decreased from 2.5-2.15 eV with increase the grain size and laser fluence. By using the reflectance and transmittance spectra, the refractive indices $(n)$, real, dielectric constant $\left(\varepsilon_{\text {Real }}\right)$ and imaginary dielectric constant $\left(\varepsilon_{\mathrm{Im}}\right)$ have been calculated and discussed graphically.
\end{abstract}

Key words: NPs, laser ablation, AFM, optical properties, transmittance spectra, nanomaterials

\section{INTRODUCTION}

Cadmium telluride is consider one of the most, promising II-VI semiconductor compounds for many, electronic and optoelectronic devices,because of its optical and transport properties (Nikale et al., 2011). Extensive research was done on CdTe thin films, mainly because its potential applications, mainly in the field of polycrystalline, thin film solar cells and also large area electronic devices like, field effect transistors, optical thin film filters, radiation detectors, nonlinear integrated optical devices, Light Emitting Diodes (LEDs) and laser hetero structures for emission in the, infrared spectral range (Khan et al., 2012). Semiconductor NPs such as CdTe have been widely studied because of their novel properties which are determined by their shape, size and surface, modification (Mntungwa et al., 2011; Saad et al., 2013). Semiconductor Nanoparticles (NPs) (or Quantum Dots, QDs) with their unique, properties such as large surface to volume ratio, good optical characteristics, high electron, transfer efficiency, biocompatibility, dimensional similarities with biological macromolecules and high surface reaction activity have experienced huge development and a great expansion of the fields of applications (Li et al., 2013a, b). Bulk CdTe possesses a direct band gap of $1.45 \mathrm{eV}$ at room temperature that is closely optimally matched to solar spectrum and thus, the absorption edge in CdTe is very sharp (Khan et al., 2012; Ban et al., 2012; Li et al., 2013a, b). Also it possesses high optical absorption coefficient $\left(>10^{4} \mathrm{~cm}^{-1}\right)$ for the, visible solar spectrum, consequently only thin film layers of few, microns thickness are needed for the, absorption of the most, of the solar spectra photons with energy higher than, the band gap, more than (90\%) of the incident spectrum is absorbed approximately at thickness; $\mu \mathrm{m}$ whereas of thickness approximately $\mu \mathrm{m}$ will absorb nearly (100\%) of the incidenttsolar radiation (Razykov et al., 2009; Alnajjar et al., 2012; Enesca et al., 2010). CdTe NPs and colloidal NPs offer a wide range of band gap energy lies in the visible spectrum due to the quantum confinement effect (Kum et al., 2008). The high Photoluminescence (PL) quantum efficiencies of CdTe NPs make it an-interesting material for use in applications such as (solar cells, LEDs, photo detectors, photoelectrochemical devices, biological labels, biosensors, etc.,) (Mntungwa et al., 2011; Semaltianos et al., 2009; Saha and Bera, 2013).

\section{MATERIALS AND METHODS}

\section{Experimental}

Laser ablation in liquid technique: The straightforward experimental setup for the ablation experiment is schematically, shown in Fig. 1. The laser light that have been used for the ablation was the second harmonic Nd:YAG laser systemmtype HUAFEI (Laser Design, Germany). The laser operates in the Q-Switch regime with a laser pulse width of $9 \mathrm{nsec}$, repetition rate of $1 \mathrm{~Hz}, \lambda=1064 \mathrm{~nm}$ wavelength with maximum energy per pulse of $1000 \mathrm{~mJ}$ and 


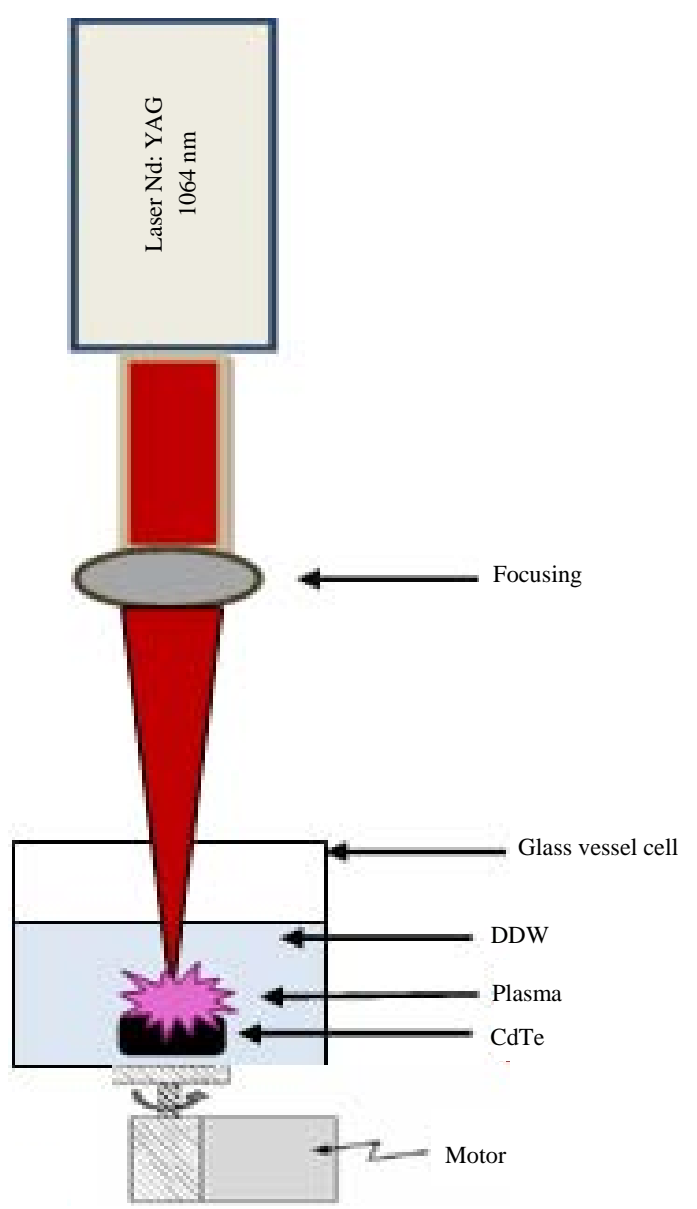

Fig. 1: Schematiccdiagram of the experimental setuppused for the synthesis of CdTe NPs suspension by laser ablation offthe target material in aaliquid environment

beam diameter of ( $4.2 \mathrm{~mm})$. The laser have been applied with a lens of $120 \mathrm{~mm}$ focal length to achieve high laser fluence.

The target bulk semiconductor which have been held inside baker glass is very small in size. The target and a baker glass are rotated byya base rotator during the process. The ablation process was typically done for (4 min) at room temperature.

Preparation of CdTe NPs colloidal suspensions: A CdTe bulk 99.999\% purity. Where it has been taken number of material bulk and then grind well in the mortar of agate for $2 \mathrm{~h}$ in order to obtain bulk material powder. After that take a sufficient amount of the powder have been compressed by piston hyderoleik USA Origin operates amounting strongly between 1-20 tons where powder press strongly the amount of 5 Tons where the target material resulting thickness was of $2 \mathrm{~mm}$ and with

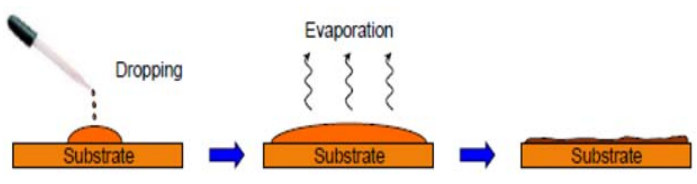

Fig. 2: Steps drop casting method

diameter circle $1 \mathrm{~cm}$. After that the target material (CdTe) has been placed on the bottom of baker and then was immersed with $3 \mathrm{~mL}$ of Double Deionized Water (DDW) with path length at $1 \mathrm{~cm}$ of laser beam above the surface of the target material. After that target material irradiation by laser with different laser energies and laser fluence, respectively 60, 100, 140, $180 \mathrm{~mJ} /$ pulse, 60, 100, 140, 180 $\mathrm{mJ} /$ pulse here number of pulses per energy ablation were constant with 300 pulse.

To avoid continuous irradiation of a single spot the target, the target and baker glass have been rotated by a base rotator during the ablation process at $20 \mathrm{rpm}$. This rotation also helps to mix the solution, homogenizing the optical path for the laser in the liquid for uniform ablation, ensuring that the nanoparticles that formed dispersed uniformly into the liquid and therefore, to reduce the agglomeration nanoparticles finally to minimize the target aging effect.

This process has been repeated three times for each ablation energy and then taken solutions colloidal of CdTe NPs suspension and placed inside plastic tubes. After that the solutions colloidal of CdTe NPs suspension were homogeneity by magnetic mixer for 45 min and then left solutions colloidal for $1 \mathrm{~h}$ in order to remove particles with sizes microscopic particles and then taken $1 \mathrm{~mL}$ of each process (experiment) ablation where it becomes prepare colloidal solution for every ablation energy of 3 $\mathrm{mL}$ of CdTe NPs Colloidal auspensions (Fig. 2).

Preparation off CdTe NPs thin film: CdTe NPs thin film hase been synthesized by deposition of CdTe NPs colloidal suspensions on glass substrates with dimensions $\left(2 \times 2 \mathrm{~cm}^{2}\right)$ with thickness $2 \mathrm{~mm}$ by drop casting method. Where that amount colloidal solution of CdTe NPs suspension that have been deposited on glass substrate was $3 \mathrm{~mL}$ for each ablation energy. The substrate temperature was at low temperature $353 \mathrm{~K}$.

Thickness measurement: The thickness of CdTe Nps thin film have been measured by using an optical interferometer method employing He-Ne laserr (wavelength of $0.632 \mu \mathrm{m}$ ). This method depends on the interference of the laser beam reflectedd from thin film surface and then substrate, the films thickness are determined using the following formula (Alias et al., 2013): 


$$
\mathrm{d}=\frac{\Delta \mathrm{x}}{\mathrm{x}} \times \frac{\lambda}{2}
$$

Where:

$$
\begin{aligned}
& \mathrm{x}=\text { The fringe width } \\
& \Delta \mathrm{x}=\text { The distance between two fringes } \\
& \lambda=\text { The wavelengthhof laser light }
\end{aligned}
$$

\section{Surface morphology measurements}

Atomic force microscope measurements: Surface morphological measurements for CdTe NPs thin films with different ablation energy have been done by SPM-AA3000 contact mode spectrometer, Angstrom Advanced IInc, company USA. Roughness and diameter of grains were obtained computerized also 2D and 3D images for all studied samples were get.

Optical measurements: The Transmittance $T(\lambda)$ of the CdTe NPs films on glass substrates has been measured in the spectral range of 300-900 nm using UV-VIS double beam spectrophotometer type UV-1800, Japanese origin, the manufacturer SHIMADZU. The Reflectance R $(\lambda)$ of NPs films has been measured in the spectral range of (400-900 nm) using spectroscopic reflectometer film thickness measurement system Made in USA, the manufacturer Angstrom Sun Technologies Inc, Model SR 300. The fundamental optical parameters like absorbance, absorption coefficient, extinction coefficient, types of transition and band gap have been calculated from transmittance spectrum. While other optical constants like refractive index (n), real dielectric constant $\left(\varepsilon_{\text {Real }}\right)$ and imaginary dielectric constant $\left(\varepsilon_{\text {Im }}\right)$ have been calculated from reflectance and transmittance spectra. The absorptionn coefficient $(\alpha)$ has been calculated by using the equation (Suhail and Ahmed, 2014):

$$
\alpha=\frac{-2.303 A}{t}
$$

where, $A=\log I_{0} / I_{t}$ and represent absorbance. The optical constants such as extinction coefficientt $(\mathrm{k})$, refractive index (n), the realland imaginary parts of dielectric constant $\left(\varepsilon_{\mathrm{r}}, \varepsilon_{\mathrm{i}}\right)$, respectively are calculated by using these equations (Suhail and Ahmed, 2014; Bakr et al., 2011; Alias et al., 2013):

$$
\mathrm{k}=\frac{\alpha \lambda}{4 \pi}
$$

Where:

$\lambda$ : The wavelength of the incident radiation

$\alpha$ : The absorption coefficient

$$
n=\left(\frac{4 R}{(1-R)^{2}}-K^{2}\right)^{\frac{1}{2}} \frac{(\mathrm{R}+1)}{(\mathrm{R}-1)}
$$

where, $\mathrm{R}$ is the reflectance:

$$
\begin{aligned}
& \varepsilon_{\mathrm{r}}=\mathrm{n}^{2}-\mathrm{k}^{2} \\
& \varepsilon_{\mathrm{i}}=2 \mathrm{nk}
\end{aligned}
$$

\section{RESULTS AND DISCUSSION}

\section{Surface morphology}

Atomic force microscope: The average grain size (grain diameter), average roughness and RMS roughness of CdTe NPs thin films prepared with different laser fluence have been measured using AFM. The results of Fig. 3 and 4 are listed in Table 1 . It is clear that the morphology of synthesized CdTe nanoparticles is strongly depended on the laser fluence. The nanoparticles prepared at low laser fluence exhibted spherical shap (ball shaped particles) while the particles synthesized with relatively high laser fluence gave elongated particles that a longed vertically as shown in 3D AFM images.

The histogram of granularity distribution of CdTe nanoparticles are given in Fig. 4. It is clear that the distribution (A, B) are very close to Gaussian distribution, i.e., taking the same distribution of laser intensity used to prepare these nanoparticles $\left(\mathrm{TEM}_{00}\right)$. The

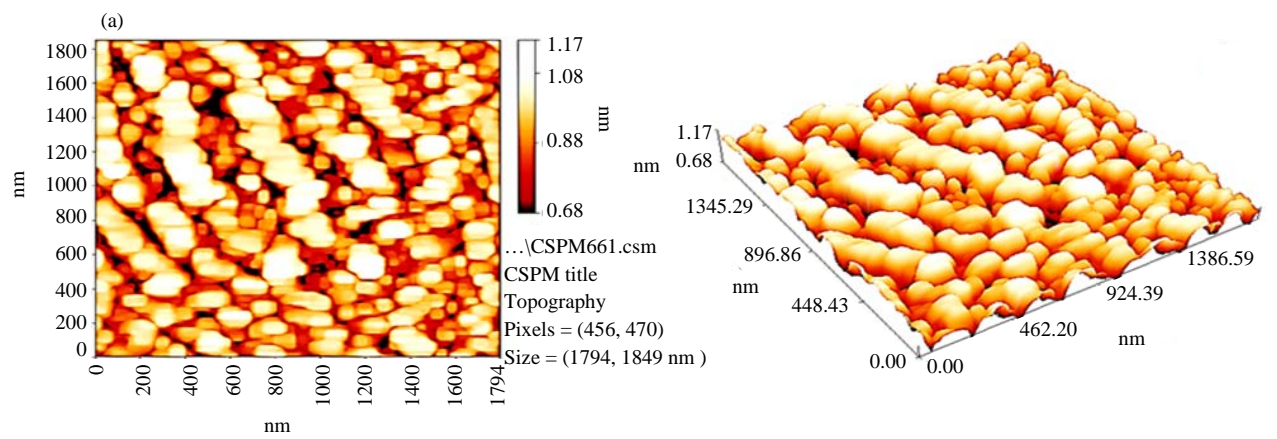

Fig. 3: Continue 

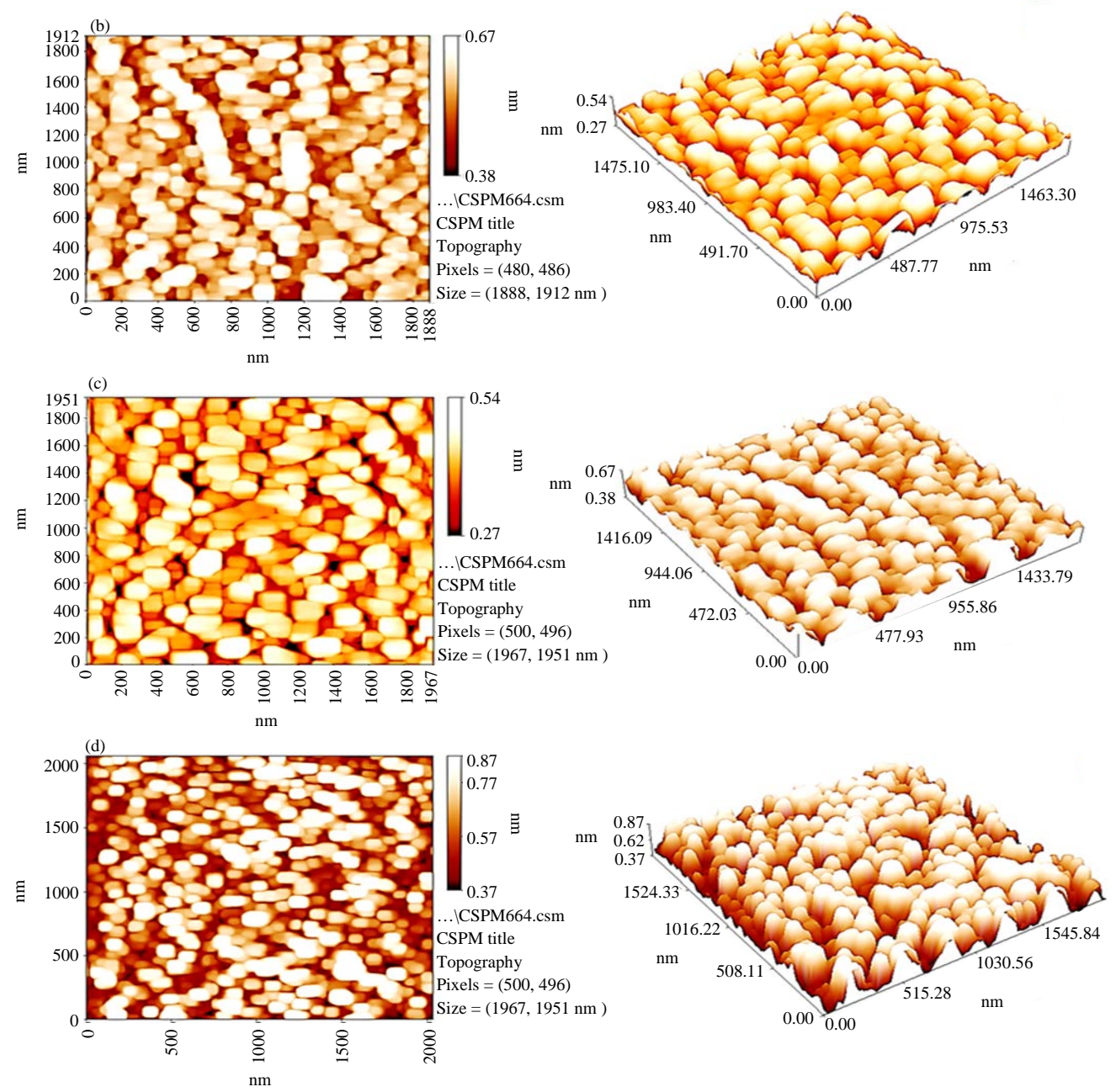

Fig. 3a-d: The AFM image results (2D and 3D) of the synthesised CdTe NPs thin films prepared with different laser fluence
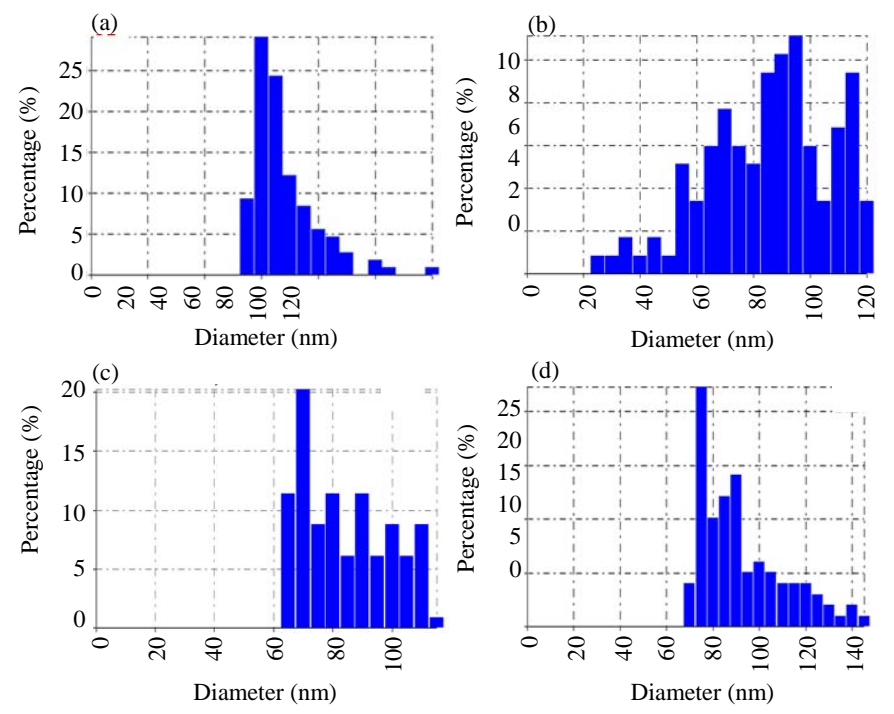

Fig. 4a-b: The histogram of granularity distribution of the synthesised CdTe NPs thin film prepared with different laser fluence; granularity cumulation distribution chart 
Table 1: AFM characteristics of the synthesized CdTe NPs thin film with different laser fluence

\begin{tabular}{lcccc}
\hline & \multicolumn{4}{c}{ AFM taken from $2 \times 2 \mu \mathrm{m}$} \\
Sample & $\begin{array}{c}\text { Laser } \\
\text { fluence } \\
\left(\mathrm{J} / \mathrm{cm}^{2}\right)\end{array}$ & $\begin{array}{c}\text { Average grain } \\
\text { size }(\mathrm{D}) \\
(\mathrm{nm})\end{array}$ & $\begin{array}{c}\text { Average } \\
\text { roughness } \\
(\mathrm{nm})\end{array}$ & $\begin{array}{c}\text { RMS } \\
\text { roughness } \\
(\mathrm{nm})\end{array}$ \\
\hline name & 0.433 & 65.42 & 0.0877 & 0.1050 \\
A & 0.722 & 70.34 & 0.0456 & 0.0556 \\
B & 1.011 & 81.85 & 0.0391 & 0.0489 \\
C & 1.299 & 90.38 & 0.0496 & 0.0635 \\
D & & & &
\end{tabular}

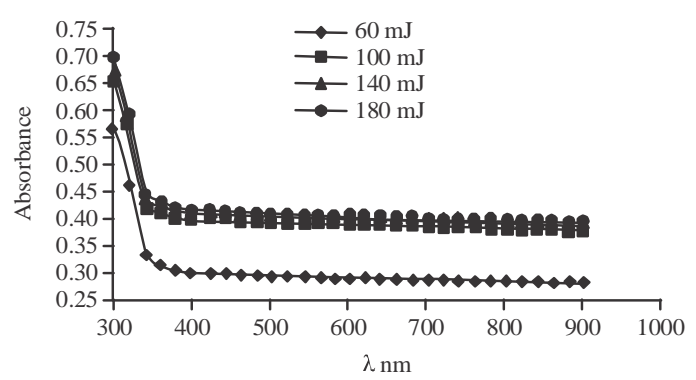

Fig. 5: The absorbance spectra versus wavelength of CdTe NPs prepared with different laser fluence

granularity distribution of CdTe NPs prepared at high laser fluence gave non-Gaussian distribution due to presence of high order laser modes, e.g., $\mathrm{TEM}_{01}$, $\mathrm{TEM}_{11}$...etc. Table 1 has showed that the average grain size increases as laser fluence increase.

\section{Optical analysis}

Absorbance (A): Figure 5 shows variation, the absorbance spectra of CdTe NPs prepared with different laser fluence as function of wavelength in the range $300-900 \mathrm{~nm}$. From the figure note that the absorbance gradually decreases with increasing wavelength also noted that the highest value for the absorbance is located in the UV region where been the approach to the value of the energy gap of CdTe NPs prepared. By watching the figure noted the blue shift of the absorbance towards highest wavelengths compared with absorption edge of bulk CdTe whenever Increased ablation energy whenever increased the grain size of CdTe NPs prepared and this is due to the emergence quantum confinement (Ruth et al., 2006). Noted that the absorbance of CdTe NPs prepared at $180 \mathrm{~mJ}$ have the highest value of the absorbance and decrease the absorbance whenever decreased ablation energy whenever decrease the grain size of CdTe NPs prepared and this is due to the energy gap of CdTe NPs prepared decrease whenever increase the grain size whenever decreased ablation energy because of the effect quantum confinement (Ruth et al., 2006). This results are conform with research (Semaltianos et al., 2009).

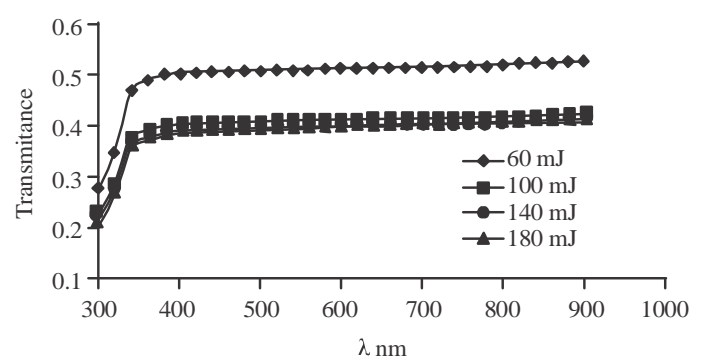

Fig. 6: The transmittance spectra versus wavelength of CdTe NPs prepared with different laser fluence

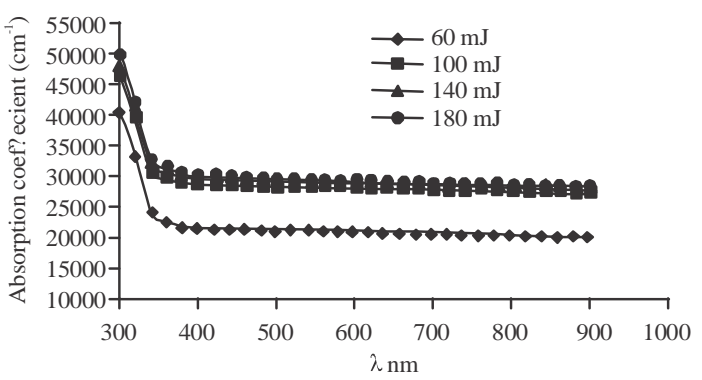

Fig. 7: The absorption coeffiecient spectra versus wavelength of CdTe NPs prepared with different laser fluence

Transmittance (T): Figure 6 shows variation the transmittance spectraaof CdTe NPs prepared with different laser fluence as function off wavelength in the range $300-900 \mathrm{~nm}$. From the the figure note that the transmittance spectra gradually increased with increasing wavelength and are roughly constant at high wavelengths and this is due to the energy gap value of nanoparticles prepared be at wavelengths few. Also noted that the spectrum transmittance of CdTe NPs prepared a few ablation energy $60 \mathrm{~mJ}$ small grain size of NPs been with higher transmittance. Addition to that whenever increase ablation energy the grain size increase the transmittance decrease and this is due to decrease the energy gap because of the effect quantum confinement (Senapati et al., 2013). This results are conform with research (Almeid et al., 2009).

Absorption coeffiecient ( $\alpha$ ): Figure 7 shows variation the absorption coeffiecient spectra of CdTe NPs prepared with different laser fluence as function of wavelength in the range $300-900 \mathrm{~nm}$. From the figure note that the absorption coefficient gradually decreases with increasing wavelength due to the decrease in absorbance as the highest absorbance of CdTe NPs prepared been of incident photons with a few wavelengths as high energies. Also noted that the blue shift of the absorption towards highest wavelengths whenever increase ablation energy 


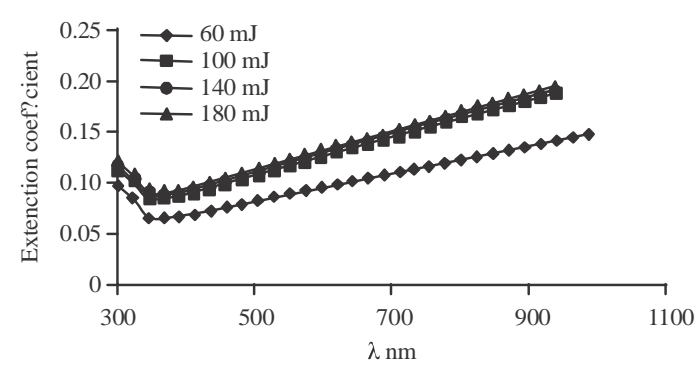

Fig. 8: The extinction coefficient spectra versus wavelength of CdTe NPs prepared with different laser fluence

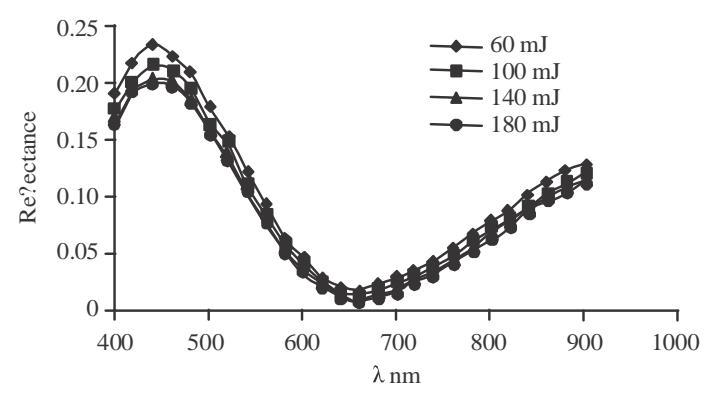

Fig. 9: The reflectance spectra versus wavelength of CdTe NPs prepared with different laser fluence

(the grain size increase) depending on the effect quantum confinement (Ruth et al., 2006). This results agree with research (Semaltianos et al., 2009).

Extinction coefficient (K): Figure 8 shows variation the extinction coefficient spectra of CdTe NPs prepared with different laser fluence as function of wavelength in the range $300-900 \mathrm{~nm}$. As the extinction coefficient decreases with increasing wavelength in the UV region and then gradually increasing at high wavelengths and this is due to the high absorbance of the CdTe NPs generated in the $\mathrm{UV}$ region and been approximately constant at high wavelengths, therefore, the extinction coefficient increases with the wavelength where been an almost linear relationship. So, it note that extinction coefficient increases with increasing energy ablation increasing grain size of the CdTe NPs generated due to increased absorption coefficient depending on the effect of quantum confinement (Ruth et al., 2006).

Reflectance (R): Figure 9 shows variation the reflectance spectra of CdTe NPs prepared with different laser fluence as function of wavelength innthe range 400-900 nm. Of the figure note that the spectra reflectance of all the preparation conditions increases at wavelengths few and starts gradually decreasing with increasing wavelength in the visible region and then gradually increase in the

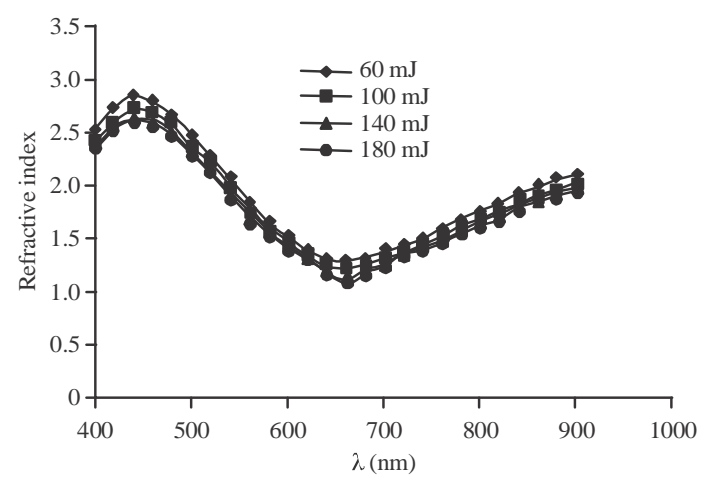

Fig. 10: The refractive index spectra versus wavelength of CdTe NPs prepared with different laser fluence

infrared region and this is due to behavior the absorbance and transmittance. Also noted that the reflectance increases as decreases energy ablation as decrease the grain size of of nanoparticles generated and this is due to increased surface area toovolume ratio which leads to increase the proportion of reflected light (Kong and Kawczak, 2008).

Refractive index (n): Figure 10 shows variation the refractive index spectra of CdTe NPs prepared with different laser fluence as function of wavelength in the range 400-900 $\mathrm{nm}$. Note from the figure that the refractiv eindex increases with the decrease grain size of nanoparticles generated as decreased energy ablation and this is due to the increased the surface area toovolume ratio and thus, increasing quantity reflected light which leads to an increase in the reflectance and thus, increase the refractive index (Kong and Kawczak, 2008). Also note that the refractive index increases in the infrared region, decrease in the visible light region and gradually increases with the wavelength at a few wavelengths.

Real part of dielectric constant $\left(\varepsilon_{\mathrm{r}}\right)$ : Figure 11 shows variation the real dielectric constant spectra of CdTe NPs prepared with different laser fluence as function of wavelength innthe range $400-900 \mathrm{~nm}$. Note change real dielectric constant of nanoparticles prepared with the wavelength where noted at a few wavelengths increases with increasing wavelength, then decrease in the visible region and then gradually increase at a high wavelengths and this is due to the behavior of the refractive index and according to the equation of real part of dielectric constant. Where note the real dielectric constant increases with decrease energy ablation (decrease grain size of CdTe NPs prepared) which leads to increase of light reflected because of the effect of quantum confinement (Ruth et al., 


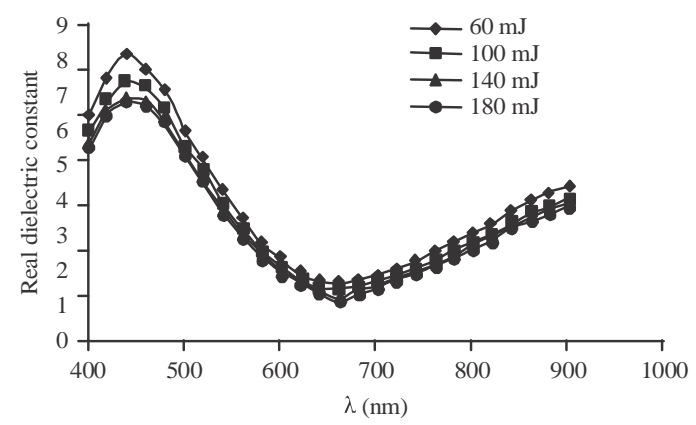

Fig. 11: The real dielectric constant spectra versus wavelength of CdTe NPs prepared with different laser fluence

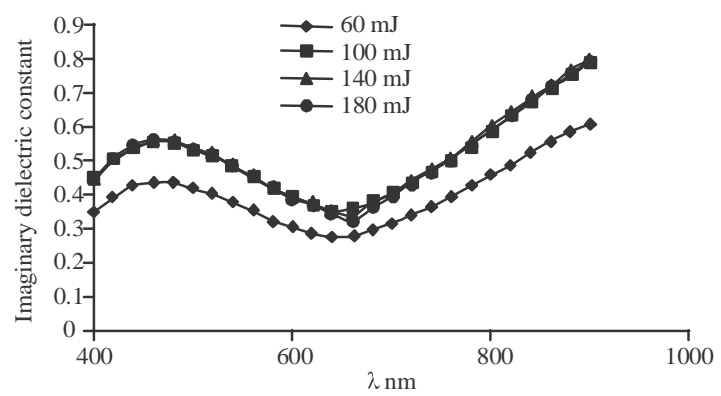

Fig. 12: The imaginary dielectric constant spectra versus wavelength of CdTe NPs prepared with different laser fluence

2006). And thus, increase the refractive index and then increases the real dielectric constant as shown in Fig. 10 and 11.

Imaginary part of dielectric constant $\left(\boldsymbol{\varepsilon}_{\mathrm{i}}\right)$ : Figure 12 shows variation the imaginary dielectric constant spectra of CdTe NPs prepared with different laser fluence as function of wavelength in the range $400-900 \mathrm{~nm}$. Note from the figure that the imaginary dielectric constant decreases with a decrease energy ablation (decrease grain size of CdTe NPs prepared) due to the decrease in energy photons falling as a result of the interaction of light with nanoparticles. In addition to an increase in the reflectance as decrease the grain size of nanoparticles prepared and therefore, the dielectric constant imaginary exhibits the behavior shown in Fig. 12 for the behavior of both the (n, k) and according to the equation of imaginary part of dielectric constant.

The optical Energy gap (Eg): The allowed direct energy gap is determineddby plotting $(\alpha h v)^{2}$ as a function of photon energyyas shown in Fig. 13 and 14. The value of the optical energy gappincrease whenever decrease energy ablation (decrease grain size of nanoparticles

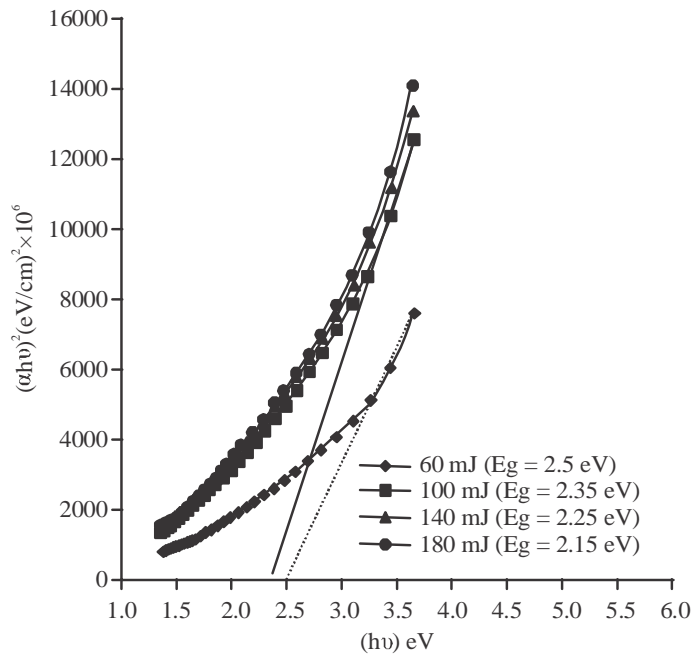

Fig. 13: The variation of $(\alpha h v)^{2}$ versus the photon energy (hv) of CdTe NPs thin films prepared with different laser fluence

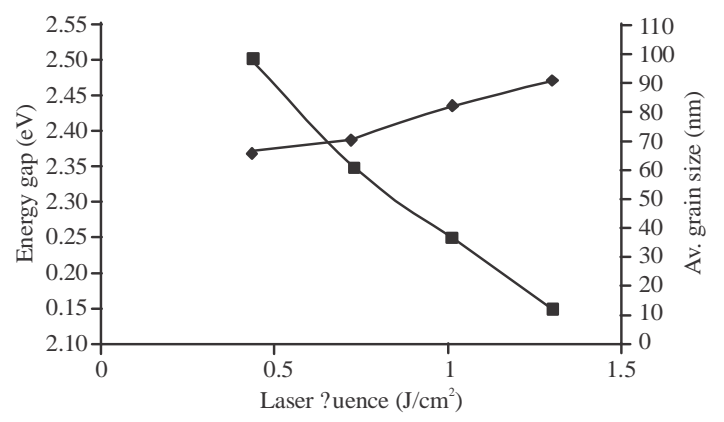

Fig. 14: The varation for energy gap and average grain size of CdTe NPs thin films as afunction of laser fluence

prepared) due to the effect of quantum confinement. Where it is whenever the energy ablation decrease decrease grain size of nanoparticles generated and thus, the number of nanoparticles increases per level increases then increased surface area to volume ratio as the same level will take a number of NPs larger than the size which leads to increased energy gap as decrease the grain size (Ruth et al., 2006). This results are conform with research (Begam et al., 2013).

\section{CONCLUSION}

Optical study has showed that the blue shift of the absorbance spectra towards highest wavelengths compared with of bulk CdTe. The possible optical transition in the CdTe NPs thin films have been found direct allowed transition. The energy gab of the synthesized CdTe Nps have been change between 
2.15-2.5 eV where the energy gap increase with decrease energy ablation (decrease grain size of nanoparticles prepared).

\section{REFERENCES}

Alias, M.F.A., R.M. Aljarrah, H. Al-Lamy and K. Adem, 2013. Investigation the effect of thickness on the structural and optical properties of nano $\mathrm{ZnO}$ films prepared by dc magnetron sputtering. Intl. J. Appl. Innovation Eng. Manage., 2: 198-203.

Almeida, D.B., E. Rodriguez, R.S. Moreira, S. Agouram and L.C. Barbosa et al., 2009. Thiol capped colloidal CdTe quantum dots synthesized using laser ablation. Nanophotonic Mater., 7393: 1-8.

Alnajjar, A.A., F.Y. Al-Shaikley and M.F.A. Alias, 2012. Optical properties and photoconductivity of undoped and in-doped CdTe thin films. J. Electron. Devices, 16: 1306-1314.

Bakr, N.A., A.M. Funde, V.S. Waman, M.M. Kamble and R.R. Hawaldar et al., 2011. Determination of the optical parameters of a-Si: $\mathrm{H}$ thin films deposited by hot wire-chemical vapour deposition technique using transmission spectrum only. Pramana, 76: 519-531.

Ban, I., M. Kristl, V. Danc, A. Danc and M. Drofenik, 2012. Preparation of cadmium telluride nanoparticles from aqueous solutions by sonochemical method. Mater. Lett., 67: 56-59.

Begam, M.R., N.M. Rao, S. Kaleemulla, M. Shobana and N.S. Krishna et al., 2013. Effect of substrate temperature on structural and optical properties of nanocrystalline CdTe thin films deposited by electron beam evaporation. J. Nano Electron. Phys., 5: 1-4.

Enesca, A., M. Comsit, I. Visa and A. Duta, 2010. New types of solid state solar cells-alternatives for silicon photovoltaic cells. Bull. Transilvania Univ. Brasov, 3: 173-182.

Khan, Z.R., M. Zulfequar and M.S. Khan, 2012. Structural, optical, photoluminescence, dielectric and electrical studies of vacuum-evaporated CdTe thin lms. Bull. Mater. Sci., 35: 169-174.

Kong, P.C. and A.W. Kawczak, 2008. Plasma synthesis of nanoparticles for nanocomposite energy applications. Proceedings of the 2008 8th World Congress on Nanocomposites, September 15-17, 2008, Crowne Plaza, San Diego, California, USA., pp: 1-12.
Kum, M.C., B.Y. Yoo, Y.W. Rheem, K.N. Bozhilov and W. Chen et al., 2008. Synthesis and characterization of cadmium telluride nanowire. Nanotechnol., 19: 1-7.

Li, D., S. Wang, J. Wang, X. Zhang and S. Liu, 2013b. Synthesis of $\mathrm{CdTe} / \mathrm{TiO}_{2}$ nanoparticles and their photocatalytic activity. Mater. Res. Bull., 48: 4283-4286.

Li, L., X. Cai, Y. Lu, Y. Ding and Y. Cheng, 2013a. Synthesis of Mn-modified CdTe nanoparticles and their application as fluorescence probe. J. Lumin., 143: 436-441.

Mntungwa, N., P.V.S. Rajasekhar and N. Revaprasadu, 2011. A facile route to shape controlled CdTe nanoparticles. Mater. Chem. Phys., 126: 500-506.

Nikale, V.M., S.S. Shinde, C.H. Bhosale and K.Y. Rajpure, 2011. Physical properties of spray deposited CdTe thin films: PEC performance. J. Semicond., 32: 1-7.

Razykov, T.M., G. Contreras-Puente, G.C. Chornokur, M. Dybjec and Y. Emirov et al., 2009. Structural, photoluminescent and electrical properties of CdTe films with different compositions fabricated by CMBD. Solar Energy, 83: 90-93.

Ruth, A.A. and J.A. Young, 2006. Generation of CdSe and CdTe nanoparticles by laser ablation in liquids. Colloids Surf. A. Physicochem. Eng. Aspects, 279: 121-127.

Saad, A.M., M.B. Mohamed, M.T.A. Kana and I.M. Azzouz, 2013. Synthesis effect, upconversion and amplified stimulated emission of luminescent CdTe NPs. Opt. Laser Technol., 46: 1-5.

Saha, S.A. and S.R. Bera, 2013. Growth and characterization of CdTe nanostructures grown by chemical reduction route. Intl. J. Metall. Mater. Sci. Eng., 3: 37-40.

Semaltianos, N.G., S. Logothetidis, W. Perrie, S. Romani and R.J. Potter et al., 2009. CdTe nanoparticles synthesized by laser ablation. Appl. Phys. Lett., 95: $1-3$.

Semaltianos, N.G., S. Logothetidis, W. Perrie, S. Romani and R.J. Potter et al., 2009. II-VI semiconductor nanoparticles synthesized by laser ablation. Appl. Phys. A., 94: 641-647.

Senapati, U.S., D.K. Jha and D. Sarkar, 2013. Green synthesis and characterization of $\mathrm{ZnS}$ nanoparticles. Res. J. Phys. Sci., 1: 1-6.

Suhail, M.H. and R.A. Ahmed, 2014. Structural, optical and electrical properties of doped copper $\mathrm{ZnS}$ thin films prepared by chemical spray pyrolysis technique. Adv. Appl. Sci. Res., 5: 139-147 